\title{
Referrals to a rheumatology unit: an evaluation of the views of patients, general practitioners, and consultants
}

\author{
D J Walker, I D Griffiths, C M Leon
}

\begin{abstract}
One hundred and twelve randomly selected patients referred to a rheumatology unit were studied, using structured questionnaires, to gain the views of patients, general practitioners (GPs), and the consultants.

There were differences in perception between these respondents on the reason for referral. Major diagnostic changes were made in less than $10 \%$ of cases. Nearly all patients claimed that some aspect of their disease had been improved as a result of the appointment.

Satisfaction with the communication aspects of the appointment contributed more to patients' overall satisfaction than did improvement in pain or disability. General practitioners' objectives were also met if communication with patients was satisfactory. In the management of chronic disease communication is important and should be recognised as such.
\end{abstract}

Audit is becoming an increasingly important aspect of medical care but presents difficulty in chronic disorders where both optimum treatment and outcome measures are ill defined. Satisfaction of both patients and general practitioners (GPs) with the service is a relevant but neglected aspect of audit in chronic disorders.

Three people are directly involved during the referral of a patient to a hospital: the GP (family doctor), the patient, and the consultant (hospital specialist). Referral of patients by their GP is a fundamental part of the National Health Service and largely controls the cost of that service. Musculoskeletal disorders are the commonest cause of physical disability within the United Kingdom, ${ }^{1}$ and it is estimated that one person in 16 consults his GP because of musculoskeletal problems each year. Most are cared for entirely by the GP, but $10 \%$ are referred to hospital where it is likely that they will attend either a rheumatology or orthopaedic clinic. There is little reported information on the subject of rheumatological referrals. One study, published in abstract form, ${ }^{2}$ found major diagnostic disagreements between GPs and hospital consultants. A further study from Sweden, ${ }^{3}$ also auditing referrals from the hospital viewpoint, again found major diagnostic disagreements. Conversely, a GP's assessment criticised hospital outpatient follow up policy, ${ }^{4}$ though not specifically rheumatological. A detailed analysis of referrals of new patients to rheumatology clinics also concludes that unnecessary follow up arrangements are common. ${ }^{5}$ We felt that a study of all aspects of patient referral involving many GPs, referring to several rather than one consultant, and including a patient evaluation of the service was required.

\section{Patients and methods}

One hundred and twelve patients referred during the previous six months were extracted randomly from the department's computer system, in the expectation that we would get about 100 replies. The routine waiting time for all of the clinics ranged between six and 12 weeks. Questionnaires were sent to the GP, the patient, and the hospital consultant involved. These questionnaires were completed independently and details of the patient were detached from the questionnaire before their return. Questionnaires were matched by study numbers only. All participants were told of this method before their completion of the questionnaire so that they would understand the anonymity of their responses.

Hospital consultants were asked to complete a questionnaire extracting the reason for the referral from the GP's letter, and expressing their degree of satisfaction with this letter. Information was sought about change in diagnosis, therapeutic interventions, and judgments of appropriateness of referral. General practitioners were asked for their objectives of the referral, to what extent these were met, and their satisfaction with the reply letter. Patients were asked why they thought they had been referred to hospital and what their main problems were at the time of referral. They were asked for their satisfaction with the speed of appointment, the explanation of their problem that they received at the hospital, how pleased they were to have attended, and what had subsequently happened to their condition. They were also asked if the appointment or any action taken from it had resulted in improvement in pain; disability; the way they thought about the problem; or the way they coped with their problem. These judgments were scored on four point scales. Finally, they were asked to make a 'free text' comment on the service.

Correlations were tested by Spearman's rank correlation.

\section{Results}

\section{COMPLETION RATES}

Of the 112 patients, 98 were referrals from GPs and 14 were consultant cross referrals. Four patients had not attended for their appointments. Three further patients could not be traced because of change of address and GP. 
Table 1 Hospital diagnoses of patients included in the study

\begin{tabular}{lcr}
\hline & Number & $\%$ \\
\hline Inflammatory polyarthritis & 18 & 16 \\
Degenerative arthritis & 32 & 29 \\
Seronegative spondarthritis & 11 & 10 \\
Soft tissue rheumatism & 17 & 15 \\
Skeletal abnormality & 3 & 3 \\
Gout & 2 & 2 \\
Non-specific pain & 11 & 10 \\
Other & 14 & 13 \\
Did not attend & 4 & 4 \\
Total & 112 & \\
\hline
\end{tabular}

Seventy nine patient replies from the 105 patients contacted $(75 \%)$ were available.

General practitioner replies were received from 93 of the 98 GP referrals (95\%). All but one of the hospital questionnaires were completed. Four consultants' clinics were covered by this study. Seventy four per cent of patients were seen by consultants and $26 \%$ by junior doctors.

DIAGNOSIS AND REASONS FOR REFERRAL

Symptoms were described and no diagnosis offered by the referring GP in 34 cases. Table 1 shows diagnoses of the patients referred. Diagnosis was changed between general practice and hospital in a minor way-for example, capsulitis of shoulder to supraspinatus tendinitis-in 21/98 (22\%) cases and in a major way-for example, wrong joint or source of pain; inflammatory to degenerative arthritis—in only $9 / 98(9 \%)$ cases.

When asked about the problem for which they had been referred, patients said that pain was the main complaint in $51(84 \%)$; disability in only two (4\%); swelling or deformity in six $(11 \%)$; and stiffness in only one case. Other responses were not in terms of symptoms.

The objectives of referral were judged by the hospital consultants based on the GP's letter, by the GPs themselves, and by the patients. They were asked to identify the main reason if more than one objective was offered. Table 2 shows the results. When specifically asked only 4/54 (7\%) patients said they had asked to be referred to hospital, yet $15 / 90$ (17\%) GPs felt they were responding to patient pressure. Hospital consultants and patients perceived that the establishment of a diagnosis was the reason for referral more frequently than the GPs.

Referrals were thought by the hospital consultant to have been very or moderately appropriate in $85 / 98(87 \%)$ cases and inappropriate in only one case.

Table 2 Primary purpose of hospital referral as judged by hospital doctors, general practitioners (GPs), and patients. ${ }^{*}$ Results are shown as number (percentage)

\begin{tabular}{lccc}
\hline Purpose & $\begin{array}{l}\text { Hospital } \\
\text { doctor }\end{array}$ & GP & Patient \\
\hline Diagnosis & $39(40)$ & $21(23)$ & $24(44)$ \\
Specific treatment & $27(28)$ & $36(40)$ & $21(39)$ \\
Long term management & $27(28)$ & $16(18)$ & $5(9)$ \\
Patient pressure & $4(4)$ & $15(17)$ & $4(7)$ \\
Other & 0 & $2(2)$ & 0 \\
Total & 97 & 90 & 54 \\
\hline
\end{tabular}

"Several patients omitted to answer this question.
Table 3 Communication between general practitioners (GPs) and hospital doctors. Results are shown as number (percentage)

\begin{tabular}{lll}
\hline $\begin{array}{l}\text { Letter } \\
\text { satisfactory }\end{array}$ & $\begin{array}{l}\text { Hospital satisfaction } \\
\text { with GP letter }\end{array}$ & $\begin{array}{l}\text { GP satisfaction } \\
\text { with hospital } \\
\text { letter }\end{array}$ \\
\hline Very & $58(62)$ & $53(65)$ \\
Moderately & $24(26)$ & $27(33)$ \\
Just & $10(11)$ & $1(1)$ \\
Not & $1(1)$ & $1(1)$ \\
Total & 93 & 82 \\
\hline
\end{tabular}

The GPs replied that in $41(81 \%)$ cases all or most of their objectives were met and in only two cases was none of their objectives met.

\section{COMMUNICATION}

Table 3 shows the judgment of the GP on the hospital letter and the hospital consultant on the GP's letter. The vast majority of both were moderately or very satisfactory.

\section{FOLLOW UP POLICY}

Forty five of 105 patients (43\%) were discharged after their first appointment, and it was expected that a further $25(24 \%)$ would receive only one follow up appointment. For 15 (14\%) a short series of appointments (three to five) was expected. It was planned that only $20(19 \%)$ would be followed up long term. This was very similar to the GP's 'expectation' but at variance with the hospital consultant's 'interpretation' of the GP's letter.

\section{ACTION TAKEN}

Hospital consultants judged that in $62 / 105$ $(59 \%)$ patients, advice was the only major action taken. Twenty five (24\%) were referred for physiotherapy, 14 (13\%) were given injections, nine $(8 \%)$ were fitted with appliances, and 15 (14\%) had a major drug intervention-for example, starting 'second line' agents or corticosteroids.

\section{PATIENT ANALYSIS OF CONSULTATION}

Table 4 shows the patients' reported improvements in pain and disability, and these are contrasted with the hospital consultant's expectation of the extent to which the patient would be helped. Pain was greatly improved in $32 / 65$ (49\%) patients. Of the remainder, an improvement in disability, coping, or perception occurred in the majority, leaving only seven patients who were not improved in any aspect of their illness. Of these, six were still pleased they had attended for their appointment.

Spontaneous comments were forthcoming from 40 patients, 11 of which were critical. In general those giving criticism did less well than those giving praise but not without exception. Criticism was of failure to influence the patient's problem in seven cases; prolonged wait for an appointment in four cases; communication problems in three cases and dissatisfaction with diagnosis; access to hospital and seeing a junior doctor in one case each. 
Table 4 Patients' reports of improvements in pain and disability resulting from their appointment, and hospital doctors' expectation of improvement. Results are shown as number (percentage)

\begin{tabular}{lclll}
\hline & Cured & A lot & A little & Not at all \\
\hline Patient reports of change in: & $22(34)$ & $10(15)$ & $14(22)$ & $19(29)$ \\
Pain* & $10(15)$ & $26(39)$ & $15(23)$ & $15(23)$ \\
Disability† & $9(13)$ & $32(44)$ & $23(32)$ & $8(11)$ \\
\hline Hospital expectation of improvement & &
\end{tabular}

*Sixty five patients replied.

tSixty six patients replied.

$\ddagger$ Based on 72 patients. was said to be changed frequently by the hospital, but it is not clear whether this was a 'fine tuning' of the diagnosis or a major difference. Forty eight per cent were said to be referred owing to patient pressure, but it is uncertain whether this was as judged by the GP or the patient, and our results suggest that the patients and GPs have different impressions. Sverdrup et al reported results from a rheumatology centre in Sweden, but only $51 \%$ of their referrals came from primary care physicians, the other $49 \%$ being cross referrals. ${ }^{3}$ They again reported a diagnostic disagreement between referring doctor and specialist in $35 \%$, which is very high in comparison with our experience. Many of the differences in diagnosis were, however, minor.

There were interesting variations in the perceptions of the reasons for referral. Hospital consultants identified the establishment of the diagnosis as the primary purpose more often than either GPs or patients, and this finding is in keeping with that of Sullivan and Hoare. ${ }^{6}$ The fact that less than $10 \%$ of diagnoses were changed in a major way supports the GPs' views. It may be that when writing a referral letter GPs feel the need to ask a respectable medical question. General practitioners felt under pressure from patients to refer for specialist opinion, yet few patients felt they had been instrumental in initiating the referral.

There have been some reports on patient follow up by hospitals, ${ }^{46}$ and this seems to depend mainly on local conditions-for example, geography, diagnosis, the confidence of local GPs in handling certain patients, and the possessiveness of consultants in retaining patients. Few patients in this study were expected to have long term follow up, and this was closely in line with GPs' expectations at referral, suggesting the service is being seen primarily as a 'consultative' service. Our follow up policy is at variance with that found by Hoare and Sullivan, ${ }^{6}$ and in part may be explained by the lower percentage of patients with rheumatoid arthritis in our study and the higher percentage seen by hospital consultants.

The responses of the patients are dependent on their memory and will, therefore, not be $100 \%$ accurate. The fact of our approach might also have increased their satisfaction.

Although we might have expected to help patients by influencing pain, disability, coping, and perception of the problem, our results show clearly that the satisfaction of patients with an appointment depended much more on communication aspects than on action taken or any of the specific outcomes. Achieving good communication with patients is, therefore, of central important in providing a rheumatological service. Thus communication skills should be highlighted in teaching and training for the treatment of chronic diseases. Communication is not solely the province of the doctors but involves other members of the health care team-in particular, nursing staff, physiotherapists, and occupational therapists. The frustration of not being able adequately to control a pathological process should not limit attempts to help patients in other ways.
Compared with the two previous studies of patient referrals to rheumatology departments, differences are apparent in our clinical practice. In the study from Bristol ${ }^{2}$ the GP's diagnosis 
Medical audit inevitably has concentrated on disorders where a clearly defined outcome-for example, death or change in laboratory parameter-can be measured in relation to treatment. Most disability in the community, however, tends to follow a subacute or chronic course and is often managed by a combination of GP and hospital outpatient service. Outcome measures in these disorders are less clearly definable. Our study suggests that the current referral patterns for the management of rheumatic disorders are seen as helpful by patients, GPs, and hospital consultants. Major criticisms of the service relate to waiting times and periodic deficiencies in communication.
We thank Miss Ann Marie McAuley for helping to organise this study and for secretarial assistance. We also thank Bencard for support for this survey and the many general practitioners and patients who collaborated in the study.

1 Physical impairment: social handicap. London: Office of Health Economics, 1977.

2 Paine T F, Dieppe P A. A study of rheumatology referrals from general practice. Br $\mathcal{F}$ Rheumatol 1987; 26 (suppl 1): 32.

3 Sverdrup B, Allebeck P, Allander E. Tentative diagnoses among referrals versus diagnoses established at the department of rheumatology. Scand $\mathcal{F}$ R heumatol 1983; 12: 377-8.

4 Marsh G N. Are follow up consultations at medical outpatient departments futile? BMF 1982; 284: 1176-7.

5 Gibson T, Clark B. Simple analgesic use in rheumatoid arthritis. Ann Rheum Dis 1985; 44: $27-9$.

6 Sullivan F M, Hoare T M. New referrals to rheumatology clinics - why do they keep coming back? $\mathrm{Br} \mathcal{F}$ Rheumatol 1990; 29: 53-7. 